\title{
DREI THESEN ZUR LITERATURDIDAKTIK IM FREMDSPRACHENUNTERRICHT
}

\author{
KOEN VANHAEGENDOREN \\ Universität Thessalien (Griechenland) \\ margvhaeg@hotmail.com
}

\section{ZUSAMMENFASSUNG}

Dieser Beitrag plädiert für die Einbeziehung von Trivialliteratur in den fremdsprachlichen Literaturunterricht, betont die gesellschaftliche Relevanz des Literaturunterrichts und versteht dessen soziale Funktion als durchaus komplementär zur sprachlichen Komponente im breiteren Rahmen der interkulturellen Kommunikation.

SCHLAGWORTE: Literaturunterricht: Fremdsprachenunterricht; Deutsch als Fremdsprache: Trivialliteratur; ästhetische Erziehung; Erziehung zur Emanzipation; gesellschaftsbezogene I iteraturdidaktik; interkulturelle Kommunikation.

\section{RESUMEN}

La presente contribución defiende el uso de la Literatura popular en la enseñanza de la literatura en lengua extranjera, acentúa la relevancia social en la enseñanza de la Literatura y entiende su función social como oportuno complemento de los los elementos lingüísticos en el marco más amplio de ls comunicación intercultural.

Palabras Clave: Enseñanza de la Literatura; enseñanza de lenguas extranjeras; el alemán como lengua extranjera; I iteratura popular; educación estética; educación para la emancipación: enseñanza sociológica de la Literatura: comunicación intercultural. 
Wenn im Titel das Wort «Thesen» benutzt wird, sind damit keine nie dagewesenen Erkenntnisse gemeint. Vielmehr ist folgender Beitrag der Versuch einer Verteidigung schon bekannter Standpunkte, die offenbar immer noch nicht allgemein anerkannt sind, wie plausibel sie auch sein mögen.

\section{1. «EINE AUSWEITUNG Des LiteraturbegrifFS TUT NOT»}

Literatur wird seit langem ${ }^{1}$ pädagogisch gebraucht - manchmal auch mißbraucht, denn auch die Literatur ist ein Mittel, durch das Individuum und Gesellschaft erzogen und gebildet werden können. Was wird aber unter Literatur verstanden, wenn man sie pädagogisch einsetzt? Über das literarische Phänomen bestehen unterschiedliche Auffassungen, die im folgenden knapp wiedergegeben werden. Literatur (vom lateinischen littera, «Buchstabe») bedeutet etymologisch «Schrifttum», alles Geschriebene, und umfaßt den gesamten Bestand von allen möglichen Schriftwerken, also z. B. auch Bücher wissenschaftlicher Art über alle Gebiete oder etwa Artikel zur Literaturdidaktik im Fremdsprachenunterricht. Im engeren Sinn bedeutet Literatur nur die sogenannte schöne Literatur, also Belletristik, die nicht zweckgebundene Mitteilung von Gedanken und Wissen ist, sondern sich durch eine besondere ästhetische Gestaltung des «Rohstoffs» auszeichnet, im Prinzip allerdings unabhängig vom behandelten Gegenstand. Die Sprache wird in der so verstandenen Literatur zum Kunstwerk ${ }^{2}$.

Nicht nur gibt es unterschiedliche Literaturdefinitionen, sondern auch unterschiedliche Meinungen über den Inhalt oder die Inhalte der Literatur. Im Laufe der Zeit ist eine Auseinandersetzung entstanden, in der versucht wird, den Inhalt von Literatur festzulegen. So wird zwischen «wertvoller» und «minderwertiger»" «anspruchsvoller» und "weniger anspruchsvoller» oder «hoher» und «niederer» Literatur unterschieden ${ }^{4}$. Zwei Jahrhunderte lang wurde gegen die sogenannte Trivialliteratur polemisiert ${ }^{5}$. So lagen denn auch für lange Zeit «weniger anspruchsvolle» literarische

' Ein Beispiel genüge: In der griechisch-römischen Antike spielte die Literatur eine außerordentliche Rolle in der Erziehung. Zahllose Generationen von Griechen wurden mit den alten Dichtern großgezogen. So konnte ein bekannter Forscher des 20. Jh. in seiner Geschichte der Erziehung in der Antike Platons Wort von Homer als «Erzieher Griechenlands» übernehmen; vgl. Marrou, Henri-Irénée: Histoire de l'éducation dans l'Antiquité, 2 Bde, Paris 1981, Bd. 1. Le monde grec, S. 33 (auch S. 244-245).

2 Vgl. Wahrig, Gerhard: Deutsches Wörterbuch mit einem "Lexikon der deutschen Sprachlehre", Berlin München - Wien 1975, Sp. 2367 und Wilpert, Gero von: Sachwörterbuch der Literatur (Kröners Taschenausgabe, 231), Stuttgart 1955, S. 314.

${ }^{3}$ Kreft, Jürgen: Grundprobleme der Literaturdidaktik. Eine Fachdidaktik im Konzept sozialer und individueller Entwicklung und Geschichte (Uni-Taschenbücher, 714), Heidelberg 1977, S. 197-198 gibt einen Überblick über die Kriterien, die die «höhere» von der «minderwertigen» Literatur unterscheiden, und spricht über die elaborierte Sprache der Hochliteratur einerseits und über die Kulturindustrie und bestimmte «bürgerliche» Auffassungen, die die Trivialliteratur widerspiegelt, andererseits.

${ }_{4}^{4}$ Vgl. Wilkending, Gisela: Ansätze zur Didaktik des Literaturunterrichts. Darstellung - Analyse (Literaturund Forschungsberichte zur Pädagogik, 3), Weinheim - Basel ${ }^{4}$ 1976, S. 54-57.

${ }^{3}$ Eine Geschichte dieser Polemik und des Hintergrunds ihrer ästhetischen Wertvorstellungen seit dem Ende des 18. Jh. findet man bei Schulte-Sasse, Jochen: Die Kritik an der Trivialliteratur seit der Aufklärung. Studien zur Geschichte des modernen Kitschbegriffs (Bochumer Arbeiten zur Sprach- und Literaturwissenschaft, 6), München 1971. 
Texte, die nur als Unterhaltungsliteratur für den größeren Teil der Gesellschaft betrachtet wurden, außerhalb des Forschungsinteresses ${ }^{6}$. Allerdings ist diese starke Polarisierung der Literatur von mehreren Literaturkritikern - u. a. Malte Dahrendorf - angegriffen worden. Dahrendorf spricht über die Diskriminierung einer Literatur, die unter dem Begriff "Trivialliteratur» verachtet wird. Die Ablehnung der Trivialliteratur ist nach Dahrendorfs Auffassung offenkundig bildungspolitisch und sozial bedingt und eben daher vorurteilsverdächtig. Die übliche ästhetische Hierarchie, die die sogenannte Trivialliteratur als minderwertig abstempelt, geht sicherlich auf eine mangelnde Reflexion des eigenen sozialen Standorts zurück: In dieser Wertschätzung sagt der Forscher mehr über seine eigene soziale Position und seine sozialen Vorurteile als über etwaige Eigenschaften oder den Wert der betrachteten Texte aus? ${ }^{7}$. Aufgrund dieser Erkenntnisse wird die Beschäftigung auch mit «minderwertiger» Literatur seit drei Jahrzehnten als selbstverständlich angesehen ${ }^{8}$. Die Trivialliteratur ist zu einem bildungsrelevanten Gegenstand geworden, an dem sich u. a. literarische Erziehung im Sinne einer Hinführung zur Lesemündigkeit vollziehen kann'. Und auch im Hinblick auf die im folgenden Absatz zu erwähnende demokratische Erziehung kann Trivialliteratur einen Beitrag leisten.

\section{2. «DER LITERATURUNTERRICHT IST GESELLSCHAFTLICH RELEVANT»}

Als besondere Leistung des Menschen hat die Kunst ihre spezifischen Wirkungen, für die der Literaturunterricht den Lernenden sensibilisieren kann. Nicht nur kann die Lesefähigkeit weiterentwickelt werden, sondern auch und vor allem kann das Verständnis

6 Vgl. Klein, Albert - Hecker, Heinz: Trivialliteratur (Grundstudium Literaturwissenschaft. Hochschuldidaktische Arbeitsmaterialien, 10), Wiesbaden 1977, S. 9-12 und Bark, Joachim: «Der Kreistanz ums Triviale. Probleme der Forschung und des Unterrichts», in: Annamaria Rucktäschel - Hans Dieter Zimmermann (Hrsg.): Trivialliteratur, München 1976, S. 10-29, dort S. 10-20.

Vgl. Dahrendorf, Malte: Literaturdidaktik im Umbruch. Aufsätze zur Literaturdidaktik, Trivialliteratur, Jugendliteratur (Studienbücher Literaturwissenschaft), Düsseldorf 1975, S. 81-129. Vgl. auch die Bedenken, die ich an anderer Stelle und in einem ganz anderen Zusammenhang über die Ablehnung der sogenannten Trivialliteratur und die Hierarchisierung literarischer Werke nach ästhetischen Kriterien geäußert habe (Vanhaegendoren, Koen: «La Position de la littérature congolaise dans l'ensemble des littératures africaines: une approche du cheminement de la pensée congolaise», in: Revue luxembourgeoise de littérature générale e comparée, 2001-2002, S. 18-43, dort S. 35-37). Damit will ich nicht für eine Nivellierung aller Literatur als gleichwertig plädieren. In dieser Frage der ästhetischen Wertschätzung, die oft ideologisch vorbelastet ist, trete ich für einen gemäßigten Standpunkt ein, der neben den Werken eines großenteils berechtigten Kanons auch anderes Schriftum zuläßt, und $\mathrm{zwar}$ auch für die Unterrichtsbehandlung. Für eine ausführlichere Auseinandersetzung mit den Positionen von Dahrendorf ist hier nicht der geeignete Platz; ich verweise dafür auf die Kritik von Bredella, Lothar: Einführung in die Literaturdidaktik (Urban-Taschenbücher, 230), Stuttgart 1976, S. 52-54 an Dahrendorfs soziologischer Literaturauffassung.

${ }^{8} \mathrm{Vgl}$. etwa die Beiträge im Nachschlagewerk von Lange, Günter - Neumann, Karl - Ziesenis, Werner 2. Literaturdidaktik: Klassische Form, Trivialliteratur 1998 sowie den Aufsatz von Lange, Günter, "Trivialliteratur», in: Heckt, , Karl (Hrsg.): Deutschunterricht von A bis Z. Braunschweig 2001, S. 362-365.

9 Melzer.

45 erörtert den Stellenwert der Trivialliteratur im literarischen Unterricht und betont die Verschiebung der Funktionen der Trivialliteratur im zeitgenössischen Unterricht gegenüber dem traditionellen Literaturunterricht, in dem Trivialliteratur als negative «Kontrastliteratur» zur «hohen» Literatur gelesen wurde. 
für die literarische Qualität gefördert werden. Das eigentlich Kunstvolle am literarischen Text, was ihn von einem alltäglichen «Gebrauchstext» abhebt, soll im Unterricht herausgearbeitet werden, so da $B$ der Lernende an diesem Beispiel seinen Sinn für die Kunst als notwendigen Bestandteil seines Daseins schärft ${ }^{10}$.

Neben der ästhetischen Erziehung" gibt es aber in den modernen Zeiten immer mehr auch eine andere Art literarischer Erziehung, und zwar die Erziehung zur Emanzipation, die als Hauptziel einer «demokratischen» Erziehung angesehen werden $k^{k a n n}{ }^{12}$. Im literarischen Unterricht beinhaltet die Berücksichtigung der politischen Konsequenzen die Erziehung zur Bereitschaft, am literarischen und sozialen Leben kritisch teilzunehmen, und die Förderung der Fähigkeit, seine eigene Stellung in der Gesellschaft zu erkennen und diese gegebenenfalls zu verändern ${ }^{13}$. Wichtige Voraussetzung für ein solches gesellschaftsbezogenes Konzept des Literaturunterrichts ist die grundsätzliche Abkehr von jeder Sakralisierung von Literatur: Texte sind nicht als literarische Kleinode in der Schule zu bewahren ... Wenn die anderen Unterrichtsfächer als sozialisierend aufgefaßt werden, mit welchem Recht darf man den Literaturunterricht als isoliert von jenen übrigen Bemühungen verstehen? In Wirklichkeit gibt es, so sieht man heute ein, einen engen Zusammenhang zwischen Literatur-Umgang einerseits und der Bereitschaft und der Fähigkeit, neue Erfahrungen im persönlichen Leben wie auch in bezug auf die Außenwelt zu machen, Vorurteile abzubauen, soziale Werte nicht unkritisch zu akzeptieren, sondern sie auf ihren Sinn, ihre Funktion und ihre möglichen Folgen zu befragen, andererseits. So gesehen, kann literarische Kommunikation für die Sensibilisierung der Lernenden fruchtbar gemacht werden, die individuellen Erfahrungsmöglichkeiten als erweiterungsbedürftig anzusehen und dadurch die Notwendigkeit gesellschaftlicher Veränderungen zu erkennen ${ }^{14}$.

10 Vgl. M. Dahrendorf, a. a. O., S. 19 und 25.

11 In den sechziger Jahren stand der ästhetische Aspekt der Texte im Vordergrund: «Im Zuge der Reformierung des Literaturunterrichts konzentrierten sich die Didaktiker auf den ästhetischen Wert der Dichtung, und nicht darauf, ob die ausgewählten Stücke pädagogischen, politischen, weltanschaulichen und moralischen Intentionen gerecht werden.» (Paefgen, Elisabeth K.: Einführung in die Literaturdidaktik, Stuttgart - Weimar 1999, S. 20)

12 Dieser Anspruch wurde in den siebziger und achtziger Jahren etwa vom Bremer Kollektiv in bewuBter Konsequenz ideologiekritisch (marxistisch) fortgeführt; vgl. die Kritik von Hassenstein, Friedrich: «Literaturwissenschaft und Literaturdidaktik», in: Lange, Günter - Neumann, Karl - Ziesenis, Werner (Hrsg.): Taschenbuch des Deutschunterrichts: Grundfragen und Praxis der Sprach-und Literaturdidaktik. Baltmannsweiler 1998, S. 478-79 am Autoritären und Zweckrationalen dieser Richtung.

${ }^{13}$ In diesem Kontext sei auf das Konzept des produktions- und handlungsorientierten Literaturunterrichts hingewiesen, das in den achtziger Jahren in der Muttersprachendidaktik entwickelt wurde, aber sowohl auf den mutter- als auch auf den fremdsprachlichen Deutschunterricht sehr einfluBreich war vor. Eine Vorstellung dieses literaturdidaktischen Ansatzes mit einer Fülle von praktischen Beispielen zu lyrischen und erzählenden Texten, findet man im Buch von Haas, Gerhard: Handlungs- und produktionsorientierter Literaturunterricht. Theorie und Praxis eines ..anderen“ Literaturunterrichts für die Primar- und Sekundarstufe (Praxis Deutsch), Seelze-Velber 1997.

${ }^{4} \mathrm{Vgl}$. M. Dahrendorf, a. a. O., S. 57-59 und J. Kreft, a. a. O., S. 254-270. Letzterer betont neben den auf die Zukunft des Lemenden und der Gesellschaft bezogenen Zielen auch die Rolle des Literatur-Umgangs in der gegenwärtigen Lebenspraxis des Lemenden. 


\section{3. «DIE SOZIALE ROLLE DES FREMDSPRACHLICHEN LITERATURUNTERRICHTS} ERGÄNZT DESSEN SPRACHLICHE ASPEKTE»

Obige Ausführungen gelten allgemein für jede Art von Literaturunterricht, unabhängig von der eingesetzten Methode, aber auch unabhängig davon, ob die Sprache, in der die zu lesenden Texte geschrieben sind, Muttersprache oder Fremdsprache für den Lernenden ist. $\mathrm{Da}$ es in diesem Beitrag um Unterricht von $\mathrm{DaF}$ geht, sei in diesem Abschnitt etwas näher auf die besondere Situation des Literaturunterrichts als Teil des Fremdsprachenunterrichts eingegangen.

Eine erste Feststellung ist, da $B$ in bezug auf den Fremdsprachenunterricht der Literaturunterricht oft «nur» als Teil des Sprachunterrichtes gesehen wird und damit dem Literaturunterricht eine praktische Nuance gegeben wird. Nach dem Urteil mancher Didaktiker versteht sich dabei von selbst, daß das gesamte Ziel des Literatur- und Sprachunterrichts die Kommunikationsfähigkeit des Schülers ist ${ }^{15}$. Das Verhältnis zwischen Literaturunterricht und Deutschunterricht ist im Hinblick auf die Intentionen, Inhalte und Lernbereiche jedoch etwas komplizierter ${ }^{16}$. Wichtig erscheint mir für eine Positionsbestimmung in dieser Frage der Begriff der «interkulturellen Kommunikation». Damit wird die zu eng verstandene, rein sprachliche Kommunikation auf eine breitere Kommunikationsfähigkeit ausgeweitet, mit der der Schüler imstande sein soll, sich in der fremden Kultur zurechtzufinden. Daß Landeskunde dabei eine zentrale Rolle spielt, bedarf keiner weiteren Erklärung ${ }^{17}$. Aber auch der literarische Gehalt einer Kultur ist für das Verstehen der täglichen Wirklichkeit, besonders auch der sprachlichen, kein Luxus ${ }^{18}$. So kann beispielsweise Migrationsliteratur und vor allem Lyrik von Autoren nichtdeutscher Herkunft sinnvoll im DaF-Unterricht eingesetzt werden; sie führt in das Problemfeld ein, wie Menschen unterschiedlicher kultureller Herkunft zusammenleben, und trägt somit zu Zielsetzungen interkulturellen Lernens und interkultureller Kommunikation bei ${ }^{19}$.

Den sprachlichen Gewinn des Literaturunterrichts nehme ich ernst, und die soziale und allgemeinbildende Funktion desselben steht zum Verständnis des fremdsprachlichen Literaturunterrichts als Teil des Sprachunterrichts in keinem Widerspruch. Vielmehr geht es um eine unterschiedliche Akzentsetzung.

Wie auch immer Literatur im Fremdsprachenunterricht eingesetzt wird und mit was für Zielen auch immer, die Situation selbst von Literatur im fremdsprachendidaktischen Kontext ist heute aufs beste gesichert. Das war aber nicht immer so. Als in den sechziger

15 Vgl. G. Wilkending, a. a. O., S. 65.

is Vgl. die detaillierte Erörterung von J. Kreft, a. a. O., S. 270-297.

17 Zum Zusammenhang von Literatur und Landeskunde vgl. Bischof, Monika - Kessling, Viola - Krechel, Rüdiger: Landeskunde und Literaturdidaktik (Fernstudienangebot Germanistik des Goethe-Instituts: Deutsch als Fremdsprache, Fernstudieneinheit 3), Berlin u. a. 1999.

${ }_{18}$ Die Notwendigkeit literarischen Wissens in der interkulturellen Kommunikation zeigt Fricke, Dietmar: "Literatur: Zentrum einer interkulturellen Landeskunde. Ein Plädoyer für mehr Literatur im Französischunterricht», in: Dietmar Fricke - Albert-Reiner Glaap (Hrsg.), Literatur im Fremdsprachenunterricht - Fremdsprache im Literaturunterricht (Schule und Forschung), Frankfurt am Main 1990, S. 62-76, dort S. 6768 anschaulich auf.

${ }^{19}$ Rösch, Heidi: «Migrationslyrik im interkulturellen Deutschunterricht», in: Materialien Deutsch als Fremdsprache 46 (1997) S. 341-366. 
und siebziger Jahren die audio-orale Methode aufkam und sich großer Beliebtheit erfreute, wurde Literatur u. a. im Fremdsprachenunterricht ${ }^{20}$ radikal in Frage gestellt. In der auf Alltagssprache gerichteten Didaktik galt Literatur als zu eliminierender Störfaktor. Inzwischen hat man die Möglichkeiten der Literaturlektüre im Unterricht auch für das Erlernen einer Fremdsprache und der fremden Kultur erkannt, so daß Literatur nunmehr einen festen Platz im Curriculum hat ${ }^{21}$. Was gegebenenfalls noch Anlaß zu wissenschaftlichen Debatten gibt, sind Fragen der Verwirklichungsmöglichkeiten dieser Einsicht über den notwendigen literarischen Aspekt der Fremdsprachenpädagogik. So werden Fragen wie die Textschwierigkeit, das Sprachniveau der Texte ${ }^{2}$, die Lesekompetenz der Schüler in der Muttersprache sowie der Fremdsprache und andere mehr erörtert ${ }^{23}$. Auch wenn in jedem einzelnen Fall konkret entschieden werden muß, ob für eine bestimmte Zielgruppe und in einem gegebenen institutionellen Rahmen ein literarisches Werk im Unterricht behandelt werden kann, sind doch von Didaktikern gewisse Kriterien ausgearbeitet worden, die Allgemeingültigkeit beanspruchen dürften ${ }^{24}$. Wer neben dem sprachlichen Nutzen auch die soziale und bildende Funktion des Literaturunterrichts im Fremdsprachenunterricht erkennt und anerkennt, wird auch der Literatur eine bedeutende Rolle im weiten Feld von $\mathrm{DaF}$ zukommen lassen. S. 38.

${ }^{20}$ Auch im Fach Deutsch hatte die Literatur in jenen Zeiten einen schweren Stand; vgl. H. Melzer, a. a. O.,

21 Vgl. Rück, Heribert: «Fremdsprachenunterricht als Literaturunterricht», in: D. Fricke - A.-R. Glaap (Hrsg.), a. a. O., S. 7-20, dort S. 7.

${ }^{22}$ In seinem Beitrag «Einfache erzählende Texte im DaF-Unterricht», in: Fragezeichen. Beiträge zur Theorie und Praxis des Deutschunterrichts in Italien, 4/5 (1986) S. 68-83 arbeitet Gerhard Neuner die didaktischen und pädagogischen Qualitäten literarischer Texte für den DaF-Unterricht heraus. Mehrere Unterrichtsbeispiele demonstrieren, wie dieses Anliegen sich mit einfachen Texten in die Praxis umsetzen läBt.

23 Ibid., S. 8-9.

24 So nennt Stütz, Wolfgang: «Kriterien zur Auswahl von Literatur für die Oberstufe», in: D. Fricke - A.-R. Glaap (Hrsg.), a. a. O., S. 21-31 sprachliche, thematische und strukturelle Kriterien. 